\title{
Treinamento de Natação na Intensidade do Limiar Anaeróbio Melhora a Aptidão Funcional de Ratos Idosos
}

\section{Swimming Training at Anaerobic Threshold Intensity Improves the Functional Fitness of Older Rats}

Verusca Najara de Carvalho Cunha' Rafael Rodrigues da Cunha'

Paulo Russo Segundo ${ }^{1,2}$

Sérgio Rodrigues Moreira ${ }^{1,2}$

Herbert Gustavo Simões ${ }^{1,2}$

1. Universidade Católica de BrasíliaUCB.

2. Programa de Pós-Graduação Stricto-Senso em Educação Física. Brasília-DF, Brasil.

Endereço para correspondência: Verusca N. C. Cunha e Dr. Herbert Gustavo Simões. QS07, LT1 s/n, sala 116, bloco G - 72030-170 - Águas Claras, DF, Brasil.

E-mail: najavrusk@gmail.com e hgsimões@gmail.com

Submetido em 20/02/2008 Versão final recebida em 22/05/2008 Aceito em 05/07/2008

\begin{abstract}
RESUMO
Os efeitos do treinamento aeróbio em intensidade relativa ao limiar de lactato (LL) foram analisados em 15 ratos idosos ( 448 dias de vida). Os grupos de animais treinados $(n=9)$ e controle $(n=6)$ foram submetidos a um teste antes e após quatro semanas de treinamento. $O$ teste incremental consistiu de uma carga inicial de $1 \%$ do peso corporal e incrementos de $1 \%$ a cada três minutos, com mensurações de lactato sanguíneo para identificação do LL por inspeção visual do ponto de inflexão da curva. O programa de treinamento consistiu de 30 minutos de natação/dia, cinco dias/semana, com sobrecarga de 5\% do peso corporal (PC), ou controle sem exercício. Foi observado aumento significativo na intensidade do $L L$ após o treinamento (pré $=4,5 \pm 1,1$ vs. Pós $=5.4 \pm 0.9 \% \mathrm{PC}$ ). A carga máxima atingida ao final do teste incremental aumentou significativamente de 39,7 $\pm 7,5 \mathrm{~g}$ no pré para $48,4 \pm 10,5 \mathrm{~g}$ no pós treinamento, sem mudanças para o grupo controle ( $44,7 \pm 8$ vs. 45,3 $\pm 9,3 \mathrm{~g})$. O peso corporal do grupo treinado não apresentou diferença como resultado de quatro semanas de natação em intensidade correspondente ao LL (641,0 $\pm 62,0$ para 636,0 $\pm 72.7 \mathrm{~g} ; \mathrm{p}>0.05)$. Por outro lado, o grupo não treinado aumentou significativamente o $P C$ de $614,0 \pm 8,0$ para $643,0 \pm 74,1 \mathrm{~g}$. A carga máxima atingida expressa tanto em valores absolutos como relativos (\%PC) aumentou significativamente após o treinamento. Conclui-se que quatro semanas de treinamento de natação em intensidade correspondente ao limiar de lactato resultou em uma melhora da aptidão aeróbia e na manutenção do peso corporal em ratos idosos.
\end{abstract}

Palavras-chave: aptidão aeróbia, limiar anaeróbio, animais idosos.

\section{ABSTRACT}

The effects of aerobic training at the lactate threshold (LT) intensity were analyzed in fifteen older rats ( 448 days old). Both the trained $(n=9)$ and control $(n=6)$ groups were submitted to an incremental exercise test before and after four weeks of training. The incremental exercise test consisted of an initial load of $1 \%$ BM and $1 \%$ increments at each 3-min with blood lactate measurements. The LT was determined by visual inspection of the blood lactate breakpoint. The training program comprised of 30-min swimming/day, 5 days/week, loaded with 5\% body mass (BM), or control without exercise. Significant increase on the LT intensity after training (pre $=4.5 \pm 1.1 \mathrm{vs}$. post $=5.4 \pm 0.9 \% \mathrm{BM}$ ). The maximal workload reached at the end of incremental test increased significantly from $39.7 \pm 7.5 \mathrm{~g}$ on pre to $48.4 \pm 10.5 \mathrm{~g}$ at post training, with no changes for the control group ( $44.7 \pm 8 \mathrm{vs} .45 .3 \pm 9.3 \mathrm{~g})$. The body mass of the trained group did not change as a result of 4 weeks of swimming at LT intensity ( $641.0 \pm 62.0$ to $636.0 \pm 72.7 \mathrm{~g} ; \mathrm{p}>0.05)$. On the other hand, the untrained group increased significantly the BM from $614.0 \pm 80.0$ to $643.0 \pm 72.7 \mathrm{~g}$. The maximal workload, as expressed both in relation to absolute and relative values (i.e. \%BM) increased significantly only as a result of training. It was concluded that four weeks of swimming training at LT intensity resulted in aerobic fitness improvement and body mass maintenance of older rats.

Keywords: aerobic fitness, anaerobic threshold, aged animals.

\section{INTRODUÇÃO}

O processo de envelhecimento está associado a inúmeras modificações fisiológicas, tais como a diminuição da força(1) e da aptidão aeróbia ${ }^{(2)}$, resultando em diminuição da independência funcional e da qualidade de vida. Por outro lado, a realização crônica de exercícios físicos mostra-se como uma importante intervenção não farmacológica no tratamento e prevenção de diversas disfunções ${ }^{(3)}$, reduzindo os fatores de risco associados ao envelhecimento e resultando em aumento da qualidade e expectativa de vida ${ }^{(4)}$.
O limiar anaeróbio (Lan) é um parâmetro de aptidão aeróbia, sendo definido como a intensidade de exercício a partir da qual ocorre aumento exponencial na concentração de lactato sanguíneo $([\mathrm{Lac}])^{(5)}$.

Diversos métodos têm sido utilizados para identificar o Lan, como o limiar ventilatório(6), o limiar glicêmico(7) e o limiar de lactato $(L L)^{(5)}$. A utilização do exercício físico em intensidade correspondente ao LL tornou-se vantajosa por determinar a capacidade aeróbia sem que seja necessário atingir o consumo máximo de oxigênio(4), diminuindo-se assim os riscos cardiovasculares ${ }^{(8)}$. A resposta do lactato sanguíneo 
ao exercício tem sido considerada válida para diagnóstico da aptidão aeróbia, prescrição e acompanhamento do treinamento em humanos ${ }^{(5)}$ e $\operatorname{ratos}^{(9)}$

O treinamento aeróbio pode resultar na melhora da saúde física de indivíduos idosos, em conseqüência das diversas adaptações cardiovasculares $^{(10)}$ e neuromusculares ${ }^{(11)}$, observáveis tanto em repouso como durante o exercício. Alguns estudos têm demonstrado aumento na massa e no volume do coração, além de diminuição da pressão arterial, da freqüência cardíaca de repouso e dos níveis glicêmicos ${ }^{(12)}$ em conseqüência da sobrecarga imposta pelo exercício crônico. A prática de atividade física diminui o número de internações, doenças

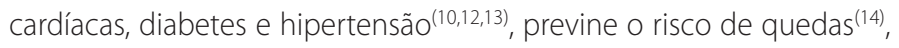
além de proporcionar bem-estar psicológico fundamental para melhor qualidade de vida ${ }^{(15)}$.

Alguns estudos, por utilizarem-se de manipulações invasivas durante exercício físico, têm sido realizados em modelo animal, envolvendo a natação ${ }^{(9,16)}$ e a corrida como forma de exercício, em ra$\operatorname{tos}^{(3,17)}$. A determinação da intensidade de exercício em ratos permite simular condições de estresse físico observadas em humanos, visando o melhor acompanhamento das alterações sistêmicas decorrentes do exercício agudo e crônico. Além disso, essa prática busca melhor compreensão dos efeitos do exercício como tratamento não medicamentoso e/ou prevenção de doenças crônicas não transmissíveis associadas ao sedentarismo e ao envelhecimento. A padronização de técnicas de avaliação da aptidão aeróbia de animais idosos é relevante. Tais técnicas permitiriam a realização de estudos sobre os efeitos de intensidades relativas de exercício nos aspectos fisiológicos associados a doenças crônicas relacionadas ao envelhecimento. Entretanto, ainda são poucos os trabalhos sobre avaliação funcional de ratos idosos, principalmente sobre os efeitos do exercício aeróbio na resposta da [Lac] e na intensidade associada ao LL nesta espécie de animais. Sendo assim, os objetivos do presente estudo foram sugerir um protocolo para determinação do LL em ratos idosos e analisar os efeitos de quatro semanas de treinamento de natação realizado em intensidade correspondente ao LL.

\section{METODOLOGIA}

\section{Amostra}

Foram utilizados ratos da linhagem Wistar $(n=15)$, machos, idosos com 15 meses de vida ${ }^{(18,19,20)}$ e sedentários com peso corporal (PC) entre 472,0 e 752,0 gramas, mantidos no Laboratório de Estudos em Educação Física e Saúde da Universidade Católica de Brasília (LEEFS) - UCB. Os animais foram alimentados com ração balanceada padrão (Purina $\left.{ }^{\circledast}\right)$ e água ad libitum e distribuídos em gaiolas coletivas. A temperatura ambiente foi controlada a $25^{\circ} \mathrm{C}$, estando o laboratório ajustado com fotoperíodo de claro e escuro de 12 horas.

\section{Grupos experimentais}

Os ratos foram divididos em dois grupos experimentais pareados por peso e idade $(p>0,05)$, sendo um grupo controle $(C)$ composto por seis ratos, com peso corporal de 614,0 \pm 83,0 que não participaram de treinamento aeróbio, e um grupo treinado ( $\mathrm{T}$ ) composto por nove ratos, com peso corporal de 641,0 \pm 62,0 gramas e que foram submetidos ao treinamento de natação.

\section{Adaptação ao meio líquido}

A adaptação consistiu em manter os ratos em contato com a água em tanques coletivos à temperatura de $30 \pm 2^{\circ} \mathrm{C}$, durante duas semanas, cinco dias por semana por 30 minutos, com o propósito de reduzir o estresse dos animais frente ao exercício físico realizado na água ${ }^{(9)}$.

\section{Avaliação da capacidade aeróbia pré e pós-treinamento}

Foram realizados testes e retestes incrementais em natação para avaliação da capacidade aeróbia. Os testes constituíram de uma sobrecarga inicial referente ao peso corporal com incrementos de 1\% da massa corporal (chumbo atado ao tórax do animal a partir de um fixador Velcro ${ }^{\oplus}$ ) a cada estágio de três minutos até a exaustão. Pausas de um minuto foram realizadas entre os estágios para troca das cargas e coleta das amostras sanguíneas.

\section{Coleta, armazenagem e dosagens sanguíneas}

Após assepsia local com álcool, foram realizadas coletas sanguíneas de $25 \mu \mathrm{l}$ de sangue retiradas da porção distal da cauda utilizando-se capilares de vidro heparinizados e calibrados. As amostras de sangue foram depositadas em microtubos (Eppendorf ${ }^{\oplus}$ ) contendo $50 \mu \mathrm{l}$ de solução de fluoreto de sódio a 1\%. A partir do método eletroenzimático em aparelho bioquímico da marca Yellow Springs Instruments-USA 2700-SELECT, as análises do lactato sanguíneo foram realizadas com participação de membrana de celulose contendo enzimas específicas que produzem peróxido de hidrogênio $\left(\mathrm{H}_{2} \mathrm{O}_{2}\right)$; ao entrar em contato com o eletrodo de platina do equipamento, a reação se processa, identificando concentração de lactato no sangue proporcional à corrente de elétrons gerada.

\section{Determinação do limiar de lactato (LL)}

A determinação do LL foi realizada a partir da inspeção visual do ponto de inflexão da curva de lactato sanguíneo vs. carga (\%), segundo técnica inicialmente proposta para humanos ${ }^{(5)}$.

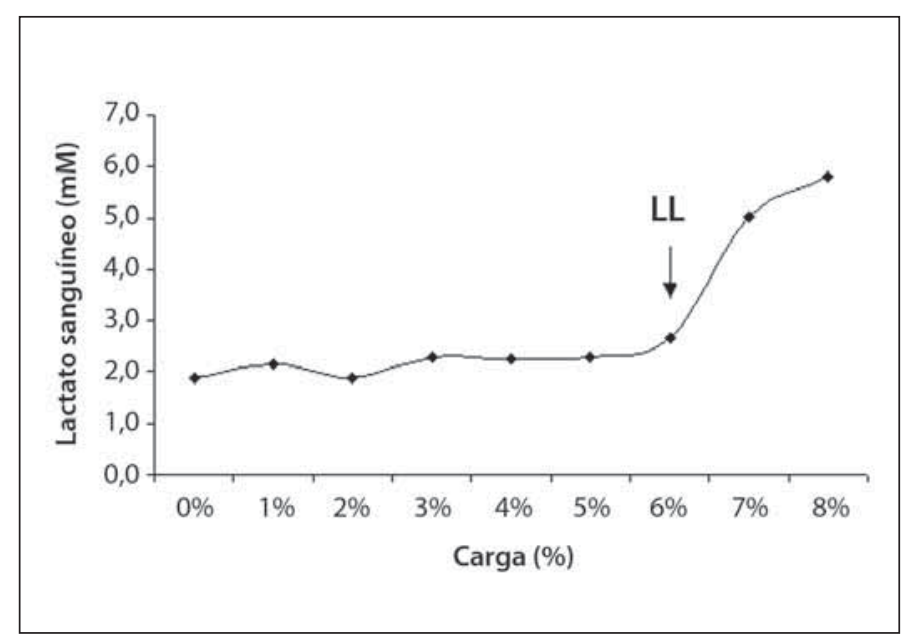

Figura 1. Exemplificação da determinação do limiar de lactato por análise de inspeção visual para um rato durante teste incremental.

\section{Protocolo de treinamento aeróbio na natação}

O protocolo de treinamento aeróbio foi realizado em tanques coletivos com água à temperatura de $30 \pm 2^{\circ} \mathrm{C}$. O treinamento foi realizado com o grupo $T$ durante quatro semanas em natação, na freqüência de cinco dias semanais com duração de 30 minutos por dia. A intensidade do treinamento foi correspondente a $5 \%$ do peso corporal, por ser intensidade sugerida como correspondente ao Lan de ratos Wistar ${ }^{(9)}$ e muito próxima da carga correspondente ao $\mathrm{LL}$ da amostra estudada. O grupo C não foi submetido ao protocolo de treinamento supracitado. 


\section{Análise estatística e procedimentos matemáticos}

Estatística descritiva, com valores de média e desvio-padrão ( \pm DP), foi aplicada. Para demonstrar as respostas relativas ao teste incremental foram selecionados momentos relativos do teste, sendo: Início (inicio do teste incremental), PM1 (ponto médio entre o início e o LL), LL (limiar de lactato), PM2 (ponto médio entre o LL e o final do teste) e Fim (exaustão do teste incremental). Teste $t$ de Student pareado foi utilizado para comparações dentro do grupo do pré para o pós-treinamento, e teste $t$ de Student não pareado quando na comparação entre grupos. O nível de significância do estudo foi de $p \leq 0,05$ (Statistica ${ }^{\oplus}$ versão 6.0).

\section{RESULTADOS}

A Tabela 1 apresenta os resultados obtidos na análise do limiar de lactato visual (LLV) da amostra avaliada.

A carga equivalente ao limiar de lactato ( $L L v g$ ) foi significativamente maior $(p<0,05)$ no grupo T pós-treinamento de quatro semanas (Tabela 1). Quando realizada comparação entre os grupos T e C para o

Tabela 1. Média ( \pm DP) da concentração de lactato sanguíneo (mM), \% da Gmáx, \%PC e gramagem (g) referente ao limiar anaeróbio identificado (LLV) no pré e póstreinamento para os grupos $\mathrm{T}$ e $\mathrm{C}$ de ratos idosos

\begin{tabular}{c|c|c|c|c}
\hline \multirow{2}{*}{} & \multicolumn{2}{|c|}{$\begin{array}{c}\text { Grupo treinado (T) } \\
(\mathbf{n = 9 )}\end{array}$} & \multicolumn{2}{c}{$\begin{array}{c}\text { Grupo controle (C) } \\
(\mathbf{n = 6 )}\end{array}$} \\
\cline { 2 - 5 } & Pré & Pós & Pré & Pós \\
\hline LLv (mM) & $2,8 \pm 0,7$ & $2,9 \pm 0,5$ & $3,9 \pm 1,2$ & $3,1 \pm 1,7$ \\
\hline $\operatorname{LLv}(\mathrm{g})$ & $28,9 \pm 6,3$ & $34,7 \pm 7,4 \neq$ & $32,8 \pm 7$ & $34,8 \pm 9,9$ \\
\hline $\operatorname{LLv}(\% \mathrm{PC})$ & $4,5 \pm 1,1$ & $5,4 \pm 0,9 \neq$ & $5,3 \pm 0,6$ & $5,3 \pm 1,1$ \\
\hline LLv (\%Gmáx) & $73,1 \pm 10,3$ & $72,2 \pm 9,4$ & $73,4 \pm 10,1$ & $75,9 \pm 7,9$ \\
\hline
\end{tabular}

LLv (mM)- concentração de lactato referente ao limiar de lactato visual; LLv (g) - carga equivalente ao limiar de lactato; LLV (\%PC) - valores percentuais do PC em intensidades correspondentes ao limiar de lactato visual; LLv (\%Gmáx) - valores percentuais da Gmáx em intensidades correspondentes ao limiar de lactato visual; $\neq p<0,05$ em relação ao pré-T.
LLv, não houve diferença significativa, tanto nas condições pré como pós-experimento.

Quando realizadas comparações dos valores percentuais do PC em intensidades correspondentes ao LLv (LLV \%PC), observou-se que o grupo T demonstrou maiores valores após o treinamento $(p<0.05)$, o mesmo não sendo observado no grupo C (Tabela 1).

Os valores médios do PC (g) não diferiram entre si no grupo T (pré $=641,0 \pm 62,0$ vs. pós $=636,2 \pm 72,7 ; p>0,05)$, por outro lado, no grupo C essa mesma variável apresentou alterações (pré $=614,0 \pm 83,0$ vs. pós $=643,3 \pm 74,1 ; p<0,05)$.

A carga máxima absoluta (Gmáx) suportada pelos ratos T apresentou aumento quando comparada com a situação pré-treinamento (pré $=39,7 \pm 7,5$ vs. pós $=48,4 \pm 10,5 ; p<0,05)$; o mesmo não é observado no grupo C (pré $=44,7 \pm 8$ vs. pós $=45,3 \pm 9,3 ; p>0,05$ ).

Além disso, quando realizada comparação das diferenças da Gmáx entre grupos (delta\%) do pré para o pós-treinamento, o grupo T demonstrou tendência $(p=0,07)$ de aumento significativo na Gmáx (Figura 2-A).

Em adicional, quando comparadas as variações do PC (delta\% PC g) do grupo T com o grupo C, observou-se diferença significativa (Figura 2-B). Uma análise da Gmáx em função do PC (\%PC) foi realizada, onde se pode verificar aumento significativo no grupo T após quatro semanas de intervenção (pré $=6,2 \pm 1,1$ vs. pós $=7,6 \pm 1,0 ; p$ $<0,05)$. Ao contrário, ao realizar a comparação dessa variável com o grupo C, não foi observada diferença (pré $=7,3 \pm 1,0$ vs. pós $=7,0 \pm$ $0,9 ; p>0,05)$.

A diferença na Gmáx (\%PC) em valores delta do pré para o póstreinamento quando comparada entre o grupo $\mathrm{T}$ e o $\mathrm{C}$ apresentou diferenças significativas (Figura 3).

Ao analisar os momentos relativos do teste incremental do pré para o pós-treinamento, o grupo T apresentou aumento significativo na variável gramagem nos momentos PM1, LL, PM2 e Fim (Figura 4-A).

Por outro lado, quando realizada análise semelhante com a variável [Lac], verificaram-se, especialmente nos momentos supralimiares (PM2 e Fim), aumentos significativos no pós-treinamento para o grupo $T$ (Figura 4-B).
Grupo T

A

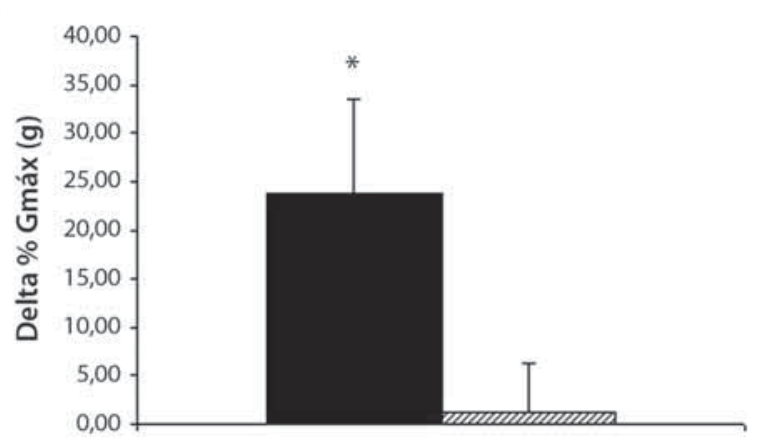

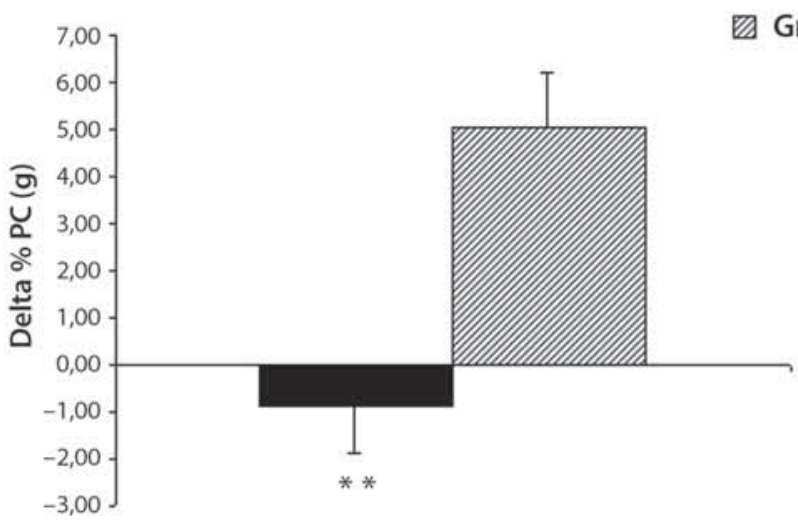

Figura 2. Média ( \pm EPM) do delta\% da Gmáx (A) e do delta\% do PC (B) nos grupos T e C de ratos idosos do pré para o pós-treinamento. ${ }^{*} p=0,07 ;{ }^{* *} p<0,05$ em relação ao grupo C. 


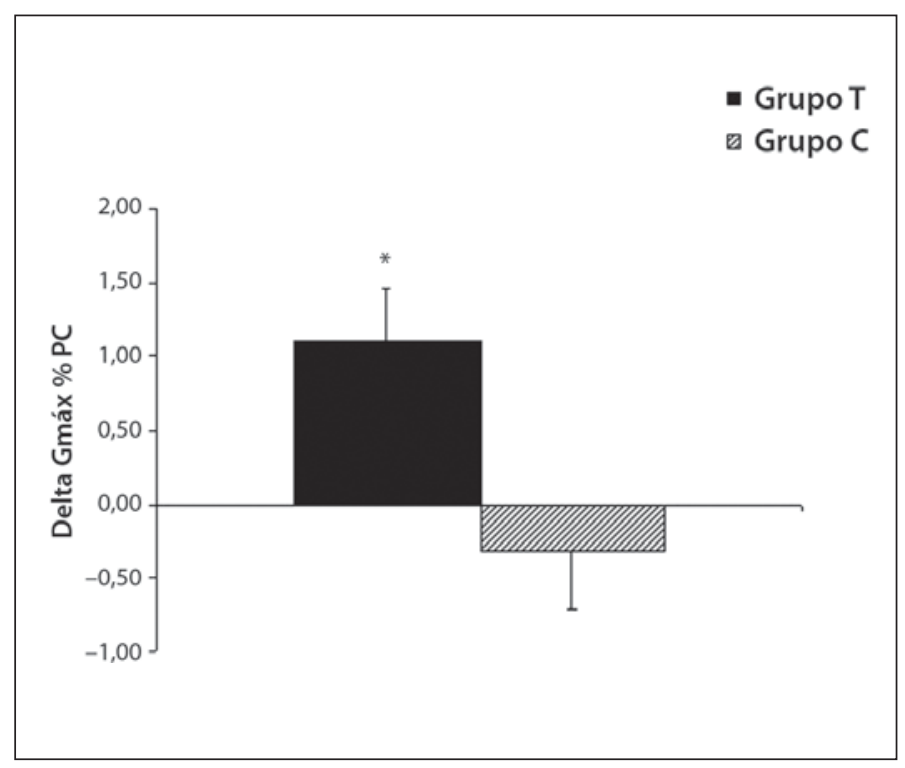

Figura 3. Média ( \pm EPM) do delta da Gmáx (\%PC) nos grupos T e C de ratos idosos do pré para o pós-treinamento. ${ }^{*} p<0,05$ em relação ao grupo $C$.

\section{DISCUSSÃO}

Os principais achados do presente estudo demonstraram melhora da aptidão funcional (nas intensidades associadas ao LL e Gmáx) e manutenção do PC de ratos idosos após quatro semanas de treinamento de natação durante 30 minutos ao dia com 5\% do PC (Tabela 1). Por outro lado, o grupo C apresentou significativo aumento $(p<0,05)$ do $P C$ no pós-experimento (pré $=614,0 \pm 83,0$ vs. pós $=643,3 \pm 74,1$; Figura 2-B), além de não modificar a capacidade funcional. Analisando o delta\% de Gmáx (Figura 2-A), tendência a diferença entre o grupo T e grupo C também fora observada $(23,7 \pm 33,5$ vs. 1,3 \pm 12,0\%; $p=0,07)$. Esses resultados possibilitaram analisar a Gmáx em função do PC (\%PC), ficando mais bem demonstrado o aumento da aptidão funcional do grupo $T$ comparado com o C (1,3 \pm 1,2 vs. $-0,3 \pm 1,0 \%$; $\mathrm{p}<0,05)$ após quatro semanas de treinamento em natação (Figura 3). Esses resultados estão de acordo com os achados de Oliveira et al. ${ }^{(12)}$, investigando o efeito de seis semanas de treinamento de natação em ratos adultos diabéticos, evidenciando o efeito do treinamento de natação no aumento da potência aeróbia, entretanto, no presente estudo em ratos idosos.
A

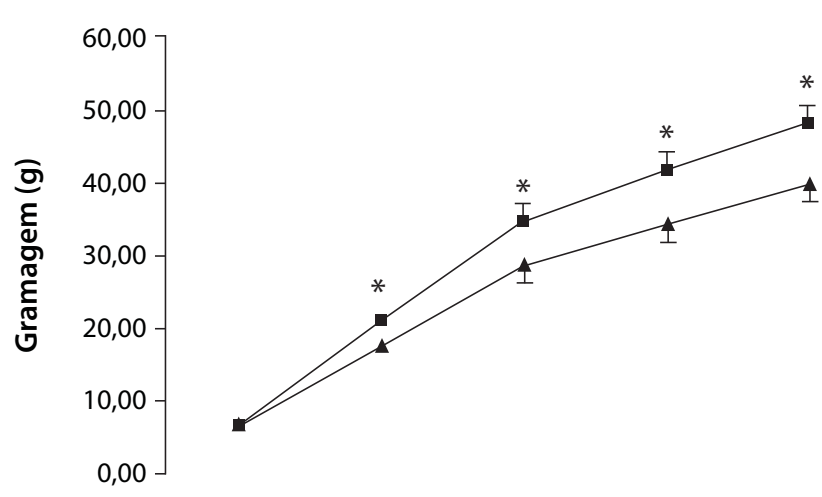

C

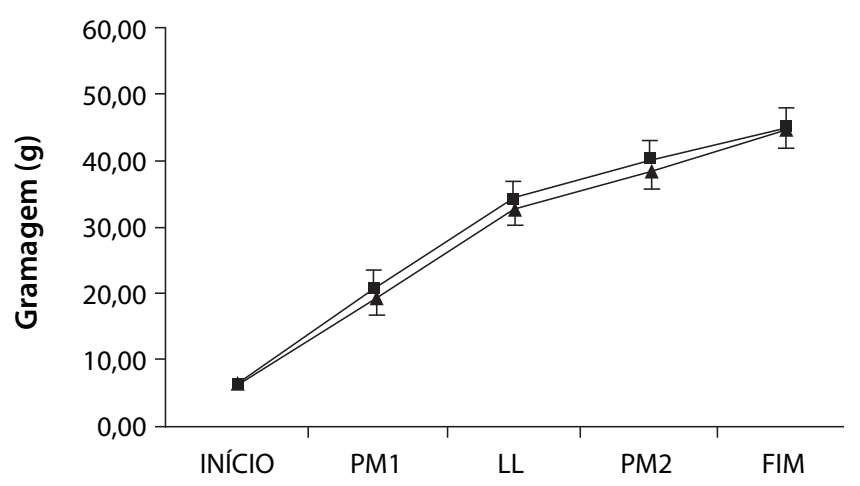

Momentos relativos
B

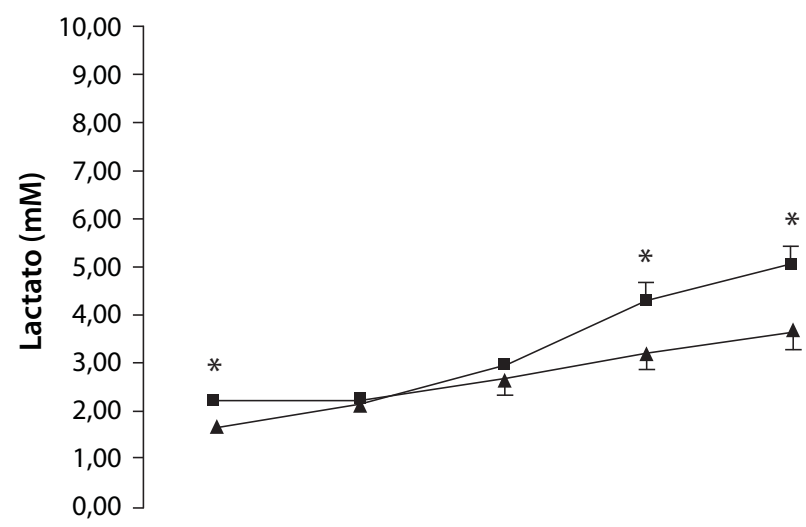

D

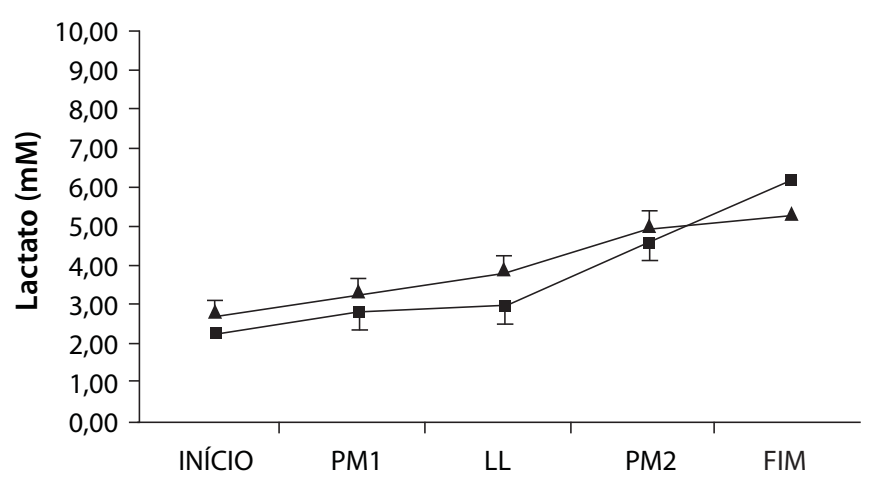

Momentos relativos

Figura 4. Média ( \pm EPM) dos momentos relativos do teste incremental nos grupos $T(A)$ e $C$ (C) para gramagem e grupos $T$ (B) e C (D) para lactato sanguíneo (linhas com triângulos $=$ pré- $T$; linhas com quadrados $=$ pós-T). ${ }^{*} p<0,05$ em relação ao grupo T pré. 
Gobatto et al. ${ }^{(9)}$, investigando ratos adultos jovens não treinados, demonstraram que cargas entre 5 e $6 \%$ do PC representavam um equilíbrio entre a produção e a remoção da [Lac], sendo consideradas intensidades com predomínio do componente aeróbio. No presente estudo observou-se resultado semelhante para $L L$ de ratos idosos, além de ter sido possível submeter a amostra estudada a intensidades de treinamento correspondentes a 5\% do PC, durante 30 minutos de exercício por dia num período de quatro semanas.

Ao analisar a [Lac] pré e pós experimento, não se observou diferença significativa tanto no grupo T como no C (Tabela 1). Entretanto, quando analisadas as variáveis LLg e LL\%PC, nota-se que o grupo T apresentou aumentos significativos do pré para o pós-experimento; por outro lado, o grupo C não obteve alterações nestes resultados (Tabela 1), sugerindo aumento absoluto e relativo da capacidade aeróbia no grupo T de ratos idosos. Outros autores ${ }^{(12)}$, ao investigar os efeitos do treinamento de natação em intensidade correspondente ao LL em ratos adultos diabéticos, encontraram resultados positivos, como no presente estudo. Esses achados identificam que o treinamento aeróbio pode proporcionar, também em animais idosos, alterações fisiológicas tais como o aumento da densidade capilar e mitocondrial, além da capacidade oxidativa enzimática muscular ${ }^{(21)}$, favorecendo a melhora da aptidão aeróbia.

Voltarelli et al. ${ }^{(16)}$, investigaram os efeitos do treinamento de quatro semanas de natação em ratos jovens em duração semelhante à do presente estudo. Esses autores observaram melhora na intensidade correspondente ao LL em sua amostra (4,9 vs. 5,4\%PC; $p<0,05)$, resultado esse semelhante ao do presente estudo, onde se verificou aumento significativo da intensidade de $L L(4,5 \pm 1,1$ vs. 5,4 \pm 0,9\%PC; $\mathrm{p}<0,05)$ em ratos idosos após quatro semanas de treinamento.

No presente estudo observou-se que o grupo $T$ de ratos idosos, após o período de experimento, apresentou maiores [Lac] em intensidades supralimiares (Figura 4-B), sugerindo a possibilidade de maior participação de unidades motoras de contração rápida quando comparado com o grupo $C$ nos mesmos momentos relativos estudados (Figura 4-D). A maior produção de [Lac] em intensidades supralimiares pode estar associada a aumentada velocidade da glicólise anaeróbia nessas intensidades após período de treinamento, efeito não identificado no grupo de ratos não treinados. Korhonen et al. ${ }^{(22)}$ analisaram o efeito do envelhecimento na resposta de [LaC] em atletas de diferentes idades durante corridas de 100 a 400 metros. Esses autores observaram que a partir dos 70 anos de idade ocorreu diminuição significativa na [Lac] durante as provas selecionadas, sugerindo possível redução na massa muscular com o processo envelhecimento, como evidenciado por Trappe et al. ${ }^{(23)}$, explicada especialmente por uma atrofia das fibras de contração rápida, seguida de redução da produção de energia proveniente do metabolismo anaeróbio e conseqüente deterioração da performance anaeróbia, o qual não ocorreu em ratos idosos após período de treinamento, como se verificou nas intensidades acima do $L L$ onde ocorreu aumento da gramagem (Figura 4-A) associado a maior lactatemia (Figura 4-B).

A literatura tem demonstrado a contribuição do exercício físico no tratamento de diversas patologias, como o diabetes tipo $2^{(24)}$, a hipertensão arterial ${ }^{(25)}$ e disfunções cardiovasculares ${ }^{(26)}$; todas apresentam íntima relação com aumento no PC e conseqüente obesidade ${ }^{(27)}$.

No presente estudo observou-se no grupo C aumento significativo do PC após quatro semanas (pré $=614,0 \pm 83,0$ vs. pós $=643,3 \pm 74,1$; $p<0,05$ ), indicando que a falta de exercício físico sistematizado favorece o ganho de PC a curto e médio prazo, enquanto que o treinamento resultou em controle de $\mathrm{PC}$ durante o envelhecimento em ratos. Apesar de não termos avaliado os diferentes componentes da composição corporal na amostra estudada, especula-se que esse ganho de PC no grupo C esteja associado ao aumento da massa gorda, a qual apresenta inversa correlação com o LL em humanos ${ }^{(28)}$, expondoos aos diversos fatores de risco relacionados à obesidade. Por outro lado, o grupo de ratos idosos após quatro semanas de treinamento em natação apresentou manutenção do PC, podendo-se constatar diferença significativa (Figura 2-B) quando comparado o delta\% de PC do grupo T com o grupo C (-0,8 $\pm 2,3$ vs. 5,0 $\pm 2,8 \% ; p<0,05)$.

Braga et al.(29), em estudos sobre exercício físico e obesidade em ratos adultos jovens, observaram redução do PC de grupos exercitados quando comparados com grupos sedentários. A manutenção e a redução do $P C$, respectivamente em ratos idosos no presente estudo e ratos adultos jovens no estudo de Braga et al. ${ }^{(29)}$, podem estar sendo explicadas por um desequilíbrio entre lipólise e lipogênese ${ }^{(30,31)}$, com conseqüente maior potencial de gasto energético em decorrência do exercício realizado.

Uma das limitações do presente estudo foi a ausência na mensuração do máximo estado estável de lactato sanguíneo (MEEL), que reflete o padrão ouro na identificação do Lan. Entretanto, o protocolo aplicado foi eficiente nessa determinação (Lan) que, segundo alguns autores ${ }^{(5)}$, pode ser a partir da inflexão na curva de lactato sanguíneo durante uma única sessão de exercício incremental e, conseqüentemente, demarcar a transição do metabolismo aeróbio para o anaeróbio, sugerindo assim intensidades individualizadas na prescrição do exercício físico.

Outras possíveis limitações que podem ocorrer em estudos com ratos estão relacionadas ao controle das condições experimentais (temperatura, superfície de contato e profundidade do tanque) durante a natação. Por outro lado, no presente estudo foi possível controlar algumas dessas limitações, como a temperatura da água $\left(30 \pm 2^{\circ} \mathrm{C}\right)$, o aumento da profundidade do tanque para o exercício, evitando o alcance dos animais ao fundo e a utilização de tanques com superfície lisa, impedindo o descanso dos animais durante o exercício ${ }^{(16)}$.

Concluímos que o LL pode ser identificado em ratos idosos a partir do protocolo descrito no presente estudo e que quatro semanas de treinamento de natação em intensidade correspondente ao LL possibilitou aumento da aptidão aeróbia desses animais. Em adicional, estudos analisando os efeitos de diferentes intensidades e volumes de exercício (maiores ou menores que no presente estudo) devem ser realizados em animais idosos, para melhor esclarecimento das possíveis adaptações morfofisiológicas em decorrência do treinamento aeróbio.

Todos os autores declararam não haver qualquer potencial conflito de interesses referente a este artigo. 


\section{REFERÊNCIAS BIBLIOGRÁFICAS}

1. Lexell J. Ageing and human muscle: observations from Sweden. Can J Appl Physiol 1993;18:2-18.

2. Fleg JL, Morrell CH, Bos AG, Brant $L$, Talbot LA, Wright JG, et al. Accelerated Longitudinal Decline of aerobic capacity in healthy older adults. Circulation 2005;112:674-82.

3. Podolin DA, Wills BK, Wood IO, Lopez M, Mazzeo RS, Roth A. Attenuation of age-related declines in glucagon-mediated signal transduction in rat liver by exercise training. Am J Physiol Endorinol Metab 2001;281:516-23.

4. American College of Sports Medicine, Mazzeo RS, Cavanagh P, et al. ACSM position stand Exercise and Physical Activity for Older Adults. Med Sci Sports Exerc. 1998;30.

5. Svedahl K, Maclntosh BR. Anaerobic threshold: the concept and methods of measurement. Can J Appl Physiol. 2003;28:299-323.

6. Wasserman K, Whipp BJ, Koyal N, Beaver WL. Anaerobic threshold and respiratory gas exchange during exercise. J Appl Physiol 1973;35:236-43.

7. Simões HG, Campbell CS, Kushnick MR, Nakamura A, Katsanos CS, Baldissera V, et al. Blood glucose threshold and the metabolic responses to incremental exercise tests with and without prior lactic acidosis induction. Eur J Appl Physiol 2003;89:603-11.

8. Wasserman K. Anaerobic Threshold and cardiovascular function. Monaldi Arch Chest Dis 2002;58:1-5

9. Gobatto CA, Mello MAR, Sibuya CY, Azevedo JRM, Santos LA \& Kokubun E. Maximal lactate steady state in rats submitted to swimming exercise. Comp Biochem Physiol A Mol Integr Physiol 2001;130:21-7.

10. Negrão CE, Moreira ED, Santos MCLM, Farah VMA, Krieger EM. Vagal function impairment after exercise training. J Appl Physiol 1992;72:1749-53

11. Jubrias SA, Esselman PC, Price LB, Cress ME, Conley KE. Large energetic adaptations of elderly muscle to resistance and endurance training. J Appl Physiol 2001;90:1663-70.

12. Oliveira CAM, Luciano E, Marcondes MCCG, Mello MAR. Effects of swimming training at the intensity equivalent to aerobic/anaerobic metabolic transition in alloxan diabetic rats. J Diabetes Complications 2007; $21: 258-64$

13. Azevedo LF, Brum PC, Rosemblatt D, Negrão CE. Características cardíacas e metabólicas de corredores de longa distância do ambulatório de cardiologia do esporte e exercício de um hospital terciário. Arq Bras Cardiol 2007;88:17-25.

14. Barnett A, Smith B, Lord SR, Williams M, Baumand A. Community-based group exercise improves balance and reduces falls in at-risk older people: a randomised controlled trial. Age Ageing 2003;32:407-14

15. Netz Y, Wu MJ, Becker BJ, Tenenbaum G. Physical activity and psychological well-being in advanced age: a meta-analysis of intervention studies. Psychol Aging 2005;20:272-84.
16. Voltarelli FA, Mello MAR, Gobatto CA. Limiar anaeróbio determinado pelo teste de lactato mínimo em ratos: efeito dos estoques de glicogênio muscular e do treinamento físico. Rev Port Cien Desp 2004;4:16-25.

17. Carvalho JF, Masuda MO, Pompeu FAMS. Method for diagnosis and control of aerobic training in rats based on lactate threshold. Comp Biochem Physiol A Mol Integr Physiol 2005;140:409-13.

18. Talbott SM, Rothkopf MM, Shapses SA. Dietary restriction of energy and calcium alters bone turnover and density in younger and older female rats. J Nutr 1998;128:640-5.

19. Yablonka-Reuveni Z, Seger R, Rivera AJ. Fibroblast growth factor promotes recruitment of skeletal muscle satellite cells in young and old rats. J Histochem Cytochem 1999;47:23-42.

20. Thompson MM, Oyama TT, Kelly FJ, KennefickTM, Anderson S. Activity and responsiveness of the reninangiotensin system in the aging rat. Am J Physiol Regul Integr Comp Physiol 2000;279:R1787-94.

21. Coggan AR, Spina RJ, King DS, Rogers MA, Brown M, Nemeth PM, et al. Skeletal muscle adaptations to endurance training in 60-to 70-yr-old men and women. J Appl Physiol 1992;72:1780-6.

22. Korhonen MT, Suomine H, Mero A. Age and sex differences in blood lactate response to sprint running in elite master athletes. Can J Appl Physiol 2005;30:647-65.

23. Trappe T, Williams R, Carrithers J, Raue U, Esmarck B, Kjaer M, et al. Influence of age and resistance exercise on human skeletal muscle proteolysis: a microdialysis approach. J Physiol 2004;554:803-13.

24. Tokmakidis SP, Zois CE, Volaklis KA, Kotsa K, Touvra A. The effects of a combined strength and aerobic exercise program on glucose control and insulin action in women with type 2 diabetes. Eur J Appl Physiol 2004;92:437-42.

25. Martin JE, Dubbert PM, Cushman WC. Controlled trial of aerobic exercise in hypertension. Circulation 1990;81:1560-7.

26. Jónsdóttir S, Andersen KK, Sigurosson AF, Sigurosson SB. The effect of physical training in chronic heart failure. Eur J Heart Fail 2006;8:97-101

27. Rosenbaum M, Leibel RL, Hirsch J. Obesity. N Engl J Med 1997;7:396-407.

28. Moreira SR, Simões GC, Hiyane WC, Campbell CSC, Simões HG. Identificação do limiar anaeróbio em indivíduos com diabetes tipo -2 sedentário e fisicamente ativos. Rev Bras Fisioter 2007;11:289-96.

29. Braga L, Mello M, Manchado F, Gobatto C. Exercício contínuo e intermitente: efeitos do treinamento e do destreinamento sobre o peso corporal e o metabolismo muscular de ratos obesos. Rev Port Cien Desp 2006;6:160-9

30. Nicklas BJ. Effects on endurance exercise on adipose tissue. Exerc Sports Sci Rev 1997;25:77-103.

31. Polak J, Moro C, Klimcakova E, Hejnova J, Majercik M, Viguerie N, et al. Dynamic strength training improves insulin sensitivity and functional balance between adrenergic alpha $2 \mathrm{~A}$ and beta pathways ins subcutaneous adipose tissue of obese subjects. Diabetologia 2005:48:2631-40. 